\begin{tabular}{|c|l|}
\hline Title & Iridium-catalyzed hydroboration of alkenes with pinacol borane \\
\hline Author(s) & Y amamoto, Y asunori; Fujikawa, Rhy ou; Umemoto, Tomokazu; Miy aura, Norio \\
\hline Citation & $\begin{array}{l}\text { Tetrahedron, 60(47), 10695-10700 } \\
\text { https://doi.org/10.1016/.tet.2004.09.014 }\end{array}$ \\
\hline Issue Date & 2004 11-15 \\
\hline Doc URL & http://hdl.handle.net/2115/15836 \\
\hline Type & article (author version) \\
\hline File Information & TETR60-47.pdf \\
\hline
\end{tabular}

Instructions for use 


\title{
Iridium-catalyzed hydroboration of alkenes with pinacolborane
}

Yasunori Yamamoto, Rhyo Fujikawa, Tomokazu Umemoto, and Norio Miyaura*

Division of Molecular Chemistry, Faculty of Engineering,

Hokkaido University, Sapporo 060-8628, Japan

\begin{abstract}
Hydroboration of terminal and internal alkenes with pinacolborane (1.2 equivalents) was carried out at room temperature in the presence of an iridium(I) catalyst (3 mol\%). Addition of dppm (2 equivalents) to $[\operatorname{Ir}(\operatorname{cod}) \mathrm{Cl}]_{2}$ gave the best catalyst for hydroboration of aliphatic terminal and internal alkenes at room temperature, resulting in addition of the boron atom to the terminal carbon of 1-alkenes with more than $99 \%$ selectivities. On the other hand, a complex prepared from dppe (2 equivalents) and $[\operatorname{Ir}(\mathrm{cod}) \mathrm{Cl}]_{2}$ resulted in the best yields for vinylarenes such as styrene. These complexes exhibited higher levels of catalyst activity and selectivity than those of corresponding rhodium complexes.
\end{abstract}

\section{Introduction}

Hydroboration of alkenes and alkynes is the most convenient method for preparation of alkyl- and 1-alkenylboron compounds. Since most H-B reagents can be added to double or triple C-C bonds without any assistance of catalysts, ${ }^{1}$ catalyzed hydroboration did not attract much attention until Männig and Nöth reported in 1985 
that the Wilkinson complex $\left[\mathrm{RhCl}\left(\mathrm{PPh}_{3}\right)_{3}\right]$ catalyzes the addition of catecholborane to alkenes and alkynes at room temperature. ${ }^{2}$ Subsequent extensive works revealed that the catalyzed hydroboration is a more interesting strategy for accelerating the slow reaction with (dialkoxy)boranes, such as catecholborane ${ }^{3}$ and pinacolborane, ${ }^{4}$ and for achieving the different chemo-, regio-, diastereo- and enantioselectivities, relative to the uncatalyzed reaction. ${ }^{5}$ Among them, $\mathrm{RhCl}\left(\mathrm{PPh}_{3}\right)_{3}$ is the most-extensively studied catalyst for hydroboration of alkenes with catecholborane, which provides an internal hydroboration product (3) for styrene with selectivity exceeding $99 \%{ }^{6}$ Such a high internal selectivity characteristic for rhodium catalysts and vinylarenes system is accounted for by a catalytic cycle proceeding through a $\pi$-benzylrhodium intermediate. ${ }^{7}$ Thus, $[\mathrm{RhCl}(\mathrm{cod})]_{2} / 4 \mathrm{PPh}_{3},{ }^{8} \quad \mathrm{Rh}\left(\eta^{3}-2\right.$-methallyl $)(\mathrm{dppb}){ }^{7} \quad\left[\mathrm{Rh}(\operatorname{cod})_{2}\right] \mathrm{BF}_{4} / 2 \mathrm{PPh}_{3}{ }^{6}$ and $\left[\mathrm{Rh}(\mathrm{cod})_{2}\right] \mathrm{BF}_{4} / \mathrm{dppb}^{6}$ selectively gave an internal product (3), whereas other metal complexes such as $\left[\mathrm{Cp}^{*} \mathrm{IrCl}_{2}\right]_{2},{ }^{9} \mathrm{RuCl}_{2}\left(\mathrm{PPh}_{3}\right)_{4},{ }^{10} \mathrm{Cp}_{2} \mathrm{TiMe}_{2},{ }^{11}$ and $\mathrm{Cp}^{*} \mathrm{Sm}^{12}$ afforded terminal hydroboration products (2) for styrene.

Catecholborane has been used in most of the reactions studied, but pinacolborane has recently been found to be an excellent alternative because it is a more stable and an easily prepared and stored hydroboration reagent. The high stability of the resulting pinacol organoboronates to moisture and chromatography is also convenient for isolation and handling. Much bulkier pinacolborane increases the terminal selectivity for styrene due to its steric hindrance. For example, the hydroboration of styrene with pinacolborane in the presence of $\mathrm{RhCl}\left(\mathrm{PPh}_{3}\right)_{3}$ yields a mixture of 2/3=41/59, and $\mathrm{Rh}(\mathrm{CO})\left(\mathrm{PPh}_{3}\right)_{2} \mathrm{Cl}$ and $\mathrm{CpNi}\left(\mathrm{PPh}_{3}\right) \mathrm{Cl}$ selectively afford a terminal product $(2>99 \%) .{ }^{13}$ In contrast to such alterable selectivity for vinylarenes depending 
upon metal catalysts and hydroboration reagents, the boron atom is selectively added to the terminal carbon of aliphatic 1-alkenes. Representative metal complexes such as $\mathrm{Rh}\left(\mathrm{PPh}_{3}\right)_{3} \mathrm{Cl}^{14}{ }^{14}[\mathrm{Rh}(\mathrm{nbd})(\mathrm{dppb})] \mathrm{BF}_{4},{ }^{14} \mathrm{Cp} * \mathrm{Sm}(\mathrm{THF}),{ }^{12} \mathrm{SmI}_{3}{ }^{15}$ and $\mathrm{Cp}_{2} \mathrm{ZrHCl}^{16}$ have been reported to yield terminal products (2) for both catecholborane and pinacolborane. Thus, selectivity and activity of representative metal catalysts have been studied extensively; however, there is little information on the corresponding iridium complexes. ${ }^{9,14 \mathrm{~b}}$ We report here that neutral iridium(I)-phosphine complexes such as $[\operatorname{Ir}(\operatorname{cod}) \mathrm{Cl}]_{2} / 2 \mathrm{dppm}$ and $[\operatorname{Ir}(\mathrm{cod}) \mathrm{Cl}]_{2} / 2 \mathrm{dppe}$ are excellent catalysts for hydroboration of terminal and internal alkenes possessing an aliphatic or aromatic substituent on the vinylic carbon with pinacolborane (Eq. 1). Most catalysts employed were prepared in situ from an air-stable cyclooctadiene complex and a phosphine ligand since previous studies using air-sensitive $\mathrm{RhCl}\left(\mathrm{PPh}_{3}\right)_{3}$ has resulted in different regioselectivities between complexes handled under argon and air. ${ }^{17}$

$$
<<\text { Eq. } 1>>
$$

\section{Results and discussion}

\subsection{Iridium-catalyzed hydroboration of alkenes}

Various neutral and cationic rhodium(I) complexes are effective for catalyzing hydroboration of styrene and other arylethenes with catecholborane (HBcat) at room temperature. The reaction selectively provides an internal hydroboration product (3) when $\mathrm{RhCl}\left(\mathrm{PPh}_{3}\right)_{3},{ }^{8,17} \mathrm{RhCl}(\mathrm{CO})\left(\mathrm{PPh}_{3}\right)_{2}{ }^{4,13}$ and $\left[\mathrm{Rh}(\mathrm{cod})_{2}\right] \mathrm{BF}_{4} / \mathrm{dppb}^{6}$ are used. The formation of dehydrogenative coupling products (4) has been reported in the hydroboration of arylethenes with phosphine-free catalysts such as 
$\left[\mathrm{RhCl}\left(p-\mathrm{MeOC}_{6} \mathrm{H}_{4} \mathrm{CH}=\mathrm{CH}_{2}\right)_{2}\right]_{2}{ }^{18}$ and $[\mathrm{RhCl}(\mathrm{cod})]_{2}{ }^{19}$ Among the representative rhodium catalysts screened for styrene, pinacolborane (HBpin) showed a high terminal selectivity, giving 2 in the presence of $\mathrm{RhCl}(\mathrm{CO})\left(\mathrm{PPh}_{3}\right)_{2}$ (Table 1 , entry 2). ${ }^{13}$ This regioselectivity is completely opposite to that of catecholborane, which selectively provided 3 in the presence of $\mathrm{RhCl}\left(\mathrm{PPh}_{3}\right)_{3}, \quad\left[\mathrm{Rh}(\operatorname{cod})_{2}\right] \mathrm{BF}_{4} / 2 \mathrm{PPh}_{3}$ or $\left[\mathrm{Rh}(\operatorname{cod})_{2}\right] \mathrm{BF}_{4} / \mathrm{dppb}^{6,8,17}$ Other neutral and cationic rhodium complexes effective for catecholborane $^{5}$ resulted in a mixture of 2,3 and 4 or a mixture of 2 and 4 for styrene (entries 1 and 3-10). Iridium complexes have rarely been used as catalysts for hydroboration, but they are catalysts that show a high terminal selectivity in hydroboration of styrene with catecholborane..$^{9,14 b}$ Indeed, most neutral and cationic iridium-phosphine complexes mainly afforded a terminal boron product (2) also for pinacolborane (entries 12-24). Among them, a combination of $[\mathrm{IrCl}(\operatorname{cod})]_{2}$ and dppe, dppp or dppb was recognized to be the best catalyst for achieving high yields and high selectivities (entries 13-15). Analogous catalysts prepared from a cationic iridium(I) precursor also predominated the formation of $\mathbf{2}$, but they were less selective than were neutral complexes (entries 17-24).

$<<$ Table $1>>$

Effects of rhodium and iridium catalysts on hydroboration of 1-octene with pinacolborane are summarized in Table 2. In contrast to styrene, which was more prone to yield internal addition products (3) or dehydrogenative coupling products (4), all rhodium(I) and iridium(I) catalysts selectively provided a terminal hydroboration product (2) for 1-octene without accompanying $\mathbf{3}$ or $\mathbf{4}$. Addition of dppp (2 equivalents to $\left.[\mathrm{Rh}(\operatorname{cod}) \mathrm{Cl}]_{2}\right)$ afforded the best rhodium catalyst, giving $2\left(\mathrm{R}=n-\mathrm{C}_{6} \mathrm{H}_{13}\right)$ in $82 \%$ yield 
(entry 5). Among the iridium complexes examined, $[\operatorname{Ir}(\operatorname{cod}) \mathrm{Cl}]_{2}$ and dppm or dppe was recognized to be the best combination for obtaining 2, with yields of $89 \%$ and $86 \%$, respectively (entries 8 and 9).

$<<$ Table $2>>$

Iridium-catalyzed hydroboration of representative terminal alkenes are summarized in Table 3. Since pinacol alkylboronates are thermally stable and insensitive to silica gel, they were easily isolated by chromatography or Kugelrohr distillation. Addition of dppm to $[\mathrm{IrCl}(\mathrm{cod})]_{2}$ worked well for aliphatic terminal alkenes, whereas dppe was a better ligand than dppm for aromatic alkenes (entries 7-12). However, both catalysts failed to catalyze hydroboration of nitrile and pyridine derivatives in high yields due to their strong coordination ability to the metal catalysts (entries 6 and 13). It has been reported that hydroboration of the terminal double bond of 1-hexen-5-one with catecholborane is much faster than reduction of the carbonyl group in the presence of $\mathrm{RhCl}\left(\mathrm{PPh}_{3}\right)_{3}$; thus giving hydroboration product (2) and 1-hexen-5-ol in a ratio of $83: 17 .^{3}$ Such a carbonyl group also remained perfectly intact in the iridium-catalyzed hydroboration with pinacolborane (entry 2). All aliphatic and aromatic terminal alkenes selectively gave terminal products (2) even for pentafluorophenylethene (entry 11). Pentafluorophenylethene, which is inert to uncatalyzed hydroboration with $9-\mathrm{BBN}$ or $\mathrm{HBSia}_{2}$ ( $\mathrm{Sia}=1,2$-dimethylpropyl), has previously been hydroborated with catecholborane in the presence of $\mathrm{RhCl}\left(\mathrm{PPh}_{3}\right)_{3}(\mathrm{Eq}$. 2). Catecholborane predominantly afforded the internal product $(\mathbf{5} / \mathbf{6}=79 / 21)$, and bulkier pinacolborane effected to further increase the terminal product in a ratio of $\mathbf{5 / 6}=29 / 71 .^{20}$ Thus, both iridium(I)-dppm and -dppe complexes shown in entry 11 
were found to be the best catalysts for obtaining a perfect anti-Markovnikov addition product.

$$
<<\text { Table } 3 \text { and Eq. 2>> }
$$

Iridium(I)-catalyzed hydroboration of internal alkenes with pinacolborane is shown in Table 4. Hydroboration of both (E)- and (Z)-4-octene resulted in the formation of pinacol 1-alkylboronates (entries 1 and 2). The corresponding reaction of (Z)-2-butene and (Z)-1-phenylpropene also resulted in isomerization to the terminal carbon (entries 3 and 4). Such isomerization to the terminal carbon, which is popular in catalyzed hydrometallation of internal alkenes, is greatly dependent on catalysts and borane reagents employed. It has been reported that such isomerization is slow in hydroboration with catecholborane using a neutral or cationic rhodium(I) catalyst ${ }^{17}$ and that the use of much bulkier pinacolborane is more prone to afford the isomerized pinacol 1-alkylboronates ${ }^{13,16}$ (Eq. 3). The reaction also took place smoothly for cyclic alkenes such as cyclohexene and norbornene (entries 5 and 6) and for 1,1-disubstituted alkenes (entries 7 and 8). Hydroboration of trisubstituted alkenes such as 2-methyl-2-butene was very slow as was reported in related metal-catalyzed hydroboration. All attempts at finding a practical catalyst for trisubstituted alkenes failed, though a phosphine-free $[\mathrm{IrCl}(\operatorname{cod})]_{2}$ exhibited a higher level of catalyst activity than that of phosphine complexes (entry 9).

$$
<<\text { Table } 4 \text { and Eq. 3>> }
$$

\section{Experimental}

\subsection{Reagents}

Pinacolborane purchased from Aldrich was purified by distillation before use 
or it can be synthesized from $\mathrm{BH}_{3} \cdot \mathrm{SMe}_{2}(\mathrm{BMS})$ and pinacol. ${ }^{4} \mathrm{RhCl}\left(\mathrm{PPh}_{3}\right)_{3},{ }^{21}$ $\mathrm{Rh}(\mathrm{CO})\left(\mathrm{PPh}_{3}\right)_{2} \mathrm{Cl},{ }^{22}[\mathrm{RhCl}(\operatorname{cod})]_{2},{ }^{23}\left[\mathrm{Rh}(\operatorname{cod})_{2}\right] \mathrm{BF}_{4},{ }^{25}[\operatorname{IrCl}(\operatorname{cod})]_{2},{ }^{26}\left[\operatorname{Ir}(\operatorname{cod})_{2}\right] \mathrm{PF}_{6}{ }^{25}$ $\left[\operatorname{Ir}(\operatorname{cod})\left(\mathrm{PPh}_{3}\right)_{2}\right] \mathrm{PF}_{6}{ }^{26}$ and $\left[\operatorname{Ir}(\operatorname{cod})\left(\mathrm{PMePh}_{2}\right)_{2}\right] \mathrm{PF}_{6}{ }^{27}$ were prepared by the reported procedures. All phosphine ligands of dppm $\left(\mathrm{Ph}_{2} \mathrm{PCH}_{2} \mathrm{PPh}_{2}\right)$, dppe $\left(\mathrm{Ph}_{2} \mathrm{PCH}_{2} \mathrm{CH}_{2} \mathrm{PPh}_{2}\right)$, dppp $\left(\mathrm{Ph}_{2} \mathrm{PCH}_{2} \mathrm{CH}_{2} \mathrm{CH}_{2} \mathrm{PPh}_{2}\right)$, dppb $\left(\mathrm{Ph}_{2} \mathrm{P}\left(\mathrm{CH}_{2}\right)_{4} \mathrm{PPh}_{2}\right), \mathrm{PCy}_{3}(\mathrm{Cy}=$ cyclohexyl), and $\mathrm{P}(t-\mathrm{Bu})_{3}$ were commercially available.

\subsection{Iridium-catalyzed hydroboration of alkenes (Tables 3 and 5)}

The catalytic hydroboration of alkenes with pinacolborane was carried out by the following general procedure. A round-bottom flask charged with $[\operatorname{Ir}(\operatorname{cod}) \mathrm{Cl}]_{2}(0.015$ mmol, $1.5 \mathrm{~mol} \%)$ and dppm or dppe $(0.03 \mathrm{mmol})$ was flushed with argon. $\mathrm{CH}_{2} \mathrm{Cl}_{2}$ (3 $\mathrm{ml})$, pinacolborane $(1.2 \mathrm{mmol})$, and alkene $(1.0 \mathrm{mmol})$ were added successively at room temperature. The mixture was then stirred at room temperature for the period shown in Tables. The reaction was quenched with methanol $(1 \mathrm{ml})$ and water $(3 \mathrm{ml})$, the product was extracted with ether, and dried over $\mathrm{MgSO}_{4}$. Chromatography on silica gel with $\mathrm{CH}_{2} \mathrm{Cl}_{2}$ gave a pinacol 1-alkylboronate.

The spectral data of compounds synthesized in Tables 3 and 4 are followed.

3.2.1. 2-Octyl-4,4,5,5-tetrametyl-[1,3,2]-dioxaborolane (2a): ${ }^{1} \mathrm{H}$ NMR (400 MHz, $\left.\mathrm{CDCl}_{3}\right) \delta 0.77(\mathrm{t}, J=7.8 \mathrm{~Hz}, 2 \mathrm{H}), 0.87(\mathrm{t}, J=6.8 \mathrm{~Hz} 3 \mathrm{H}), 1.24(\mathrm{~s}, 12 \mathrm{H}), 1.21-1.29(\mathrm{~m}$, 10H), 1.38-1.41 (m, 2H); ${ }^{13} \mathrm{C}$ NMR (100 MHz, $\left.\mathrm{CDCl}_{3}\right) \delta 14.1,22.7,24.0,24.8,29.2$, 29.4, 31.9, 32.4, 82.8; MS (EI) m/z 41 (81), 59 (58), 69 (50), 85 (82), 129 (100), 183 (10), 225 (52), 240 (3); HRMS calcd for $\mathrm{C}_{14} \mathrm{H}_{29} \mathrm{BO}_{2} ; 240.2261$ found; 240.2265.

3.2.2. 2-(5-Oxohexyl)-4,4,5,5-tetrametyl-[1,3,2]-dioxaborolane (2b): ${ }^{1} \mathrm{H}$ NMR (400 $\left.\mathrm{MHz}, \mathrm{CDCl}_{3}\right) \delta 0.78(\mathrm{t}, 2 \mathrm{H}, J=7.8 \mathrm{~Hz}), 1.24(\mathrm{~s}, 12 \mathrm{H}), 1.30-1.45(\mathrm{~m}, 2 \mathrm{H}), 1.54-1.62(\mathrm{~m}$, 
2H), $2.13(\mathrm{~s}, 3 \mathrm{H}), 2.42(\mathrm{t}, 2 \mathrm{H}, J=7.4 \mathrm{~Hz}) ;{ }^{13} \mathrm{C} \mathrm{NMR}\left(100 \mathrm{MHz}, \mathrm{CDCl}_{3}\right) \delta 23.5,24.7$, 26.3, 29.6, 43.5, 82.8, 209.1; MS (EI) m/z 43 (100), 55 (39), 69 (15), 83 (25), 111 (12), 168 (9), 211 (1), 241 (0.2); HRMS calcd for $\mathrm{C}_{12} \mathrm{H}_{23} \mathrm{BO}_{3} ; 226.1740$ found; 226.1750.

3.2.3. 2-(4-Bromobutyl)-4,4,5,5-tetrametyl-[1,3,2]-dioxaborolane (2c): ${ }^{1} \mathrm{H}$ NMR (400 $\left.\mathrm{MHz}, \mathrm{CDCl}_{3}\right) \delta 0.80(\mathrm{t}, 2 \mathrm{H}, J=7.8 \mathrm{~Hz}), 1.25(\mathrm{~s}, 12 \mathrm{H}), 1.55(\mathrm{tt}, J=7.5,7.8 \mathrm{~Hz}, 2 \mathrm{H})$, $1.87(\mathrm{tt}, J=6.8,7.5 \mathrm{~Hz}, 2 \mathrm{H}), 3.40(\mathrm{t}, J=6.8 \mathrm{~Hz}, 2 \mathrm{H}) ;{ }^{13} \mathrm{C} \mathrm{NMR}\left(100 \mathrm{MHz}, \mathrm{CDCl}_{3}\right) \delta$ 22.7, 24.8, 33.6, 35.3, 83.0; MS (EI) m/z 41 (85), 55 (66), 69 (42), 83 (100), 96 (25), 129 (34), 163 (19), 183 (66), 247 (27), 262 (0.8); HRMS calcd for $\mathrm{C}_{10} \mathrm{H}_{20} \mathrm{BBrO}_{2}$; 262.0740 found; 262.0729.

3.2.4. 2-(3-Phenoxypropyl)-4,4,5,5-tetrametyl-[1,3,2]-dioxaborolane (2d): ${ }^{1} \mathrm{H}$ NMR $\left(400 \mathrm{MHz}, \mathrm{CDCl}_{3}\right) \delta 0.92(\mathrm{t}, J=7.8 \mathrm{~Hz}, 2 \mathrm{H}), 1.28(\mathrm{~s}, 12 \mathrm{H}), 1.90(\mathrm{tt}, J=6.7,7.8 \mathrm{~Hz}, 2 \mathrm{H})$, $3.95(\mathrm{t}, J=6.7 \mathrm{~Hz}, 2 \mathrm{H}), 6.89-6.93(\mathrm{~m}, 3 \mathrm{H}), 7.24-7.28(\mathrm{~m}, 2 \mathrm{H}) ;{ }^{13} \mathrm{C}$ NMR (100 MHz, $\left.\mathrm{CDCl}_{3}\right) \delta 23.7,24.8,69.4,83.0,114.5,120.3,129.3,159.1 ; \mathrm{MS}$ (EI) $\mathrm{m} / \mathrm{z} 41$ (49), 57 (38), 69 (24), 83 (41), 94 (100), 101 (28), 119 (19) 169 (16), 189 (33), 262 (17); HRMS calcd for $\mathrm{C}_{15} \mathrm{H}_{23} \mathrm{BO}_{3} ; 262.1740$ found; 262.1738 .

3.2.5. 2-(3-Cyanopropyl)-4,4,5,5-tetrametyl-[1,3,2]-dioxaborolane (2e): ${ }^{1} \mathrm{H}$ NMR $\left(400 \mathrm{MHz} \mathrm{CDCl}_{3}\right) \delta 0.94(\mathrm{t}, J=7.8 \mathrm{~Hz}, 2 \mathrm{H}), 1.24(\mathrm{~s}, 12 \mathrm{H}), 1.78(\mathrm{tt}, J=7.2,7.8 \mathrm{~Hz}$, 2H), $2.37(\mathrm{t}, J=7.2 \mathrm{~Hz}, 2 \mathrm{H}) ;{ }^{13} \mathrm{C} \mathrm{NMR}\left(100 \mathrm{MHz}, \mathrm{CDCl}_{3}\right) \delta 20.3,24.5,24.8,83.3$, 129.0; MS (EI) m/z 43 (100), 59 (72), 68 (36), 85 (78), 96 (81), 109 (15) 137 (19), 180 (64), 194 (4); HRMS calcd for $\mathrm{C}_{10} \mathrm{H}_{18} \mathrm{BNO}_{2} ; 195.1431$ found; 195.1429.

3.2.6. 2-(2-Phenyethyl)-4,4,5,5-tetrametyl-[1,3,2]-dioxaborolane (2f): ${ }^{1} \mathrm{H}$ NMR (400 $\left.\mathrm{MHz}, \mathrm{CDCl}_{3}\right) \delta 1.14(\mathrm{t}, J=8.1 \mathrm{~Hz}, 2 \mathrm{H}), 1.22(\mathrm{~s}, 12 \mathrm{H}), 2.75(\mathrm{t}, J=8.1 \mathrm{~Hz}, 2 \mathrm{H})$, 7.13-7.28 (m, 5H); ${ }^{13} \mathrm{C}$ NMR $\left(100 \mathrm{MHz}, \mathrm{CDCl}_{3}\right) \delta 24.8,29.9,83.1,125.5,128.0,128.1$, 
144.4; MS (EI) m/z 41 (72), 59 (33), 69 (19), 84 (100), 91 (82), 105 (40) 132 (38), 175

(17), 232 (6); HRMS calcd for $\mathrm{C}_{14} \mathrm{H}_{21} \mathrm{BO}_{2} ; 232.1635$ found; 232.1649.

3.2.7. 2-(2-(4-Methoxyphenyl)ethyl)-4,4,5,5-tetrametyl-[1,3,2]-dioxaborolane (2g): ${ }^{1} \mathrm{H}$ NMR $\left(400 \mathrm{MHz}, \mathrm{CDCl}_{3}\right) \delta 1.11(\mathrm{t}, J=8.1 \mathrm{~Hz}, 2 \mathrm{H}), 1.22(\mathrm{~s}, 12 \mathrm{H}), 2.69(\mathrm{t}, J=8.1 \mathrm{~Hz}$, 2H), $3.78(\mathrm{~s}, 3 \mathrm{H}), 6.79-6.82(\mathrm{~m}, 2 \mathrm{H}), 7.12-7.18(\mathrm{~m}, 2 \mathrm{H}) ;{ }^{13} \mathrm{C} \mathrm{NMR}\left(100 \mathrm{MHz}, \mathrm{CDCl}_{3}\right) \delta$ 24.8, 29.0, 55.2, 83.0, 113.5, 128.8, 136.5, 157.5; MS (EI) m/z 41 (53), 59 (15), 69 (10), 84 (45), 91 (15), 121 (100) 134 (46), 161 (11), 262 (14); HRMS calcd for $\mathrm{C}_{15} \mathrm{H}_{23} \mathrm{BO}_{3}$; 262.1740 found; 262.1718 .

3.2.8. 2-(2-(4-methylphenyl)ethyl)-4,4,5,5-tetrametyl-[1,3,2]-dioxaborolane (2h): ${ }^{1} \mathrm{H}$ NMR (400 MHz, $\left.\mathrm{CDCl}_{3}\right) \delta 1.12(\mathrm{t}, J=8.1 \mathrm{~Hz}, 2 \mathrm{H}), 1.23(\mathrm{~s}, 12 \mathrm{H}), 2.30(\mathrm{~s}, 3 \mathrm{H}), 2.70(\mathrm{t}$, $J=8.1 \mathrm{~Hz}, 2 \mathrm{H}), 7.05-7.12(\mathrm{~m}, 4 \mathrm{H}) ;{ }^{13} \mathrm{C} \mathrm{NMR}\left(100 \mathrm{MHz}, \mathrm{CDCl}_{3}\right) \delta$ 20.9, 24.8, 29.4, 83.0, 127.8, 128.8, 134.8, 141.3; MS (EI) m/z 41 (67), 59 (21), 69 (16), 84 (100), 105 (63), 118 (23), 146 (16), 189 (6), 246 (9); HRMS calcd for $\mathrm{C}_{15} \mathrm{H}_{23} \mathrm{BO}_{2} ; 246.1791$ found; 246.1781 .

3.2.9. 2-(2-Pentafluorophenylethyl)-4,4,5,5-tetrametyl-[1,3,2]-dioxaborolane (2i): ${ }^{1} \mathrm{H} \mathrm{NMR}\left(400 \mathrm{MHz}, \mathrm{CDCl}_{3}\right) \delta 1.10(\mathrm{t}, J=8.1 \mathrm{~Hz}, 2 \mathrm{H}), 1.23(\mathrm{~s}, 12 \mathrm{H}), 2.79(\mathrm{t}, J=8.1 \mathrm{~Hz}$, $2 \mathrm{H}) ;{ }^{13} \mathrm{C} \mathrm{NMR}\left(100 \mathrm{MHz}, \mathrm{CDCl}_{3}\right) \delta 16.9,24.7,83.3,117.1,138.0,138.6,140.5,143.7$, 146.1; MS (EI) m/z 43 (100), 59 (91), 69 (21), 85 (47), 129 (28), 181 (30) 222 (23), 307 (21), 322 (6); HRMS calcd for $\mathrm{C}_{14} \mathrm{H}_{16} \mathrm{BF}_{5} \mathrm{O}_{2} ; 322.1164$ found; 322.1185 .

3.2.10. 2-(2-(2-Naphtyl)ethyl)-4,4,5,5-tetrametyl-[1,3,2]-dioxaborolane $\quad(2 \mathrm{j}):{ }^{1} \mathrm{H}$ $\operatorname{NMR}\left(400 \mathrm{MHz}, \mathrm{CDCl}_{3}\right) \delta 1.23(\mathrm{t}, J=8.1 \mathrm{~Hz}, 2 \mathrm{H}), 1.22(\mathrm{~s}, 12 \mathrm{H}), 2.92(\mathrm{t}, J=8.1 \mathrm{~Hz}$, 2H), 7.36-7.43 (m, 3H), $7.64(\mathrm{~s}, 1 \mathrm{H}), 7.73-7.79(\mathrm{~m}, 3 \mathrm{H}) ;{ }^{13} \mathrm{C} \mathrm{NMR}\left(100 \mathrm{MHz}, \mathrm{CDCl}_{3}\right) \oint$ $24.8,30.1,83.1,124.9,125.7,125.7,127.3,127.4,127.5,127.7,131.9,133.6,142.0$; 
MS (EI) m/z 41 (50), 59 (15), 69 (14), 84 (71), 115 (37), 141 (100), 154 (69), 166 (18), 182 (18), 282 (26); HRMS calcd for $\mathrm{C}_{18} \mathrm{H}_{23} \mathrm{BO}_{2} ; 282.1791$ found; 282.1774.

3.2.11. 2-(2-(4-Prydyl)ethyl)-4,4,5,5-tetrametyl-[1,3,2]-dioxaborolane (2k): ${ }^{1} \mathrm{H}$ NMR $\left(400 \mathrm{MHz}, \mathrm{CDCl}_{3}\right) \delta 1.15(\mathrm{t}, J=8.1 \mathrm{~Hz}, 2 \mathrm{H}), 1.25(\mathrm{~s}, 12 \mathrm{H}), 2.74(\mathrm{t}, J=8.1 \mathrm{~Hz}, 2 \mathrm{H})$, 7.11-7.16 (m, 2H), 8.42-8.58 (m, 2H); ${ }^{13} \mathrm{C}$ NMR (100 MHz, $\left.\mathrm{CDCl}_{3}\right) \delta$ 24.7, 29.2, 83.3, 123.5, 149.2, 153.4; MS (EI) m/z 41 (52), 59 (59), 93 (39), 106 (29), 133 (100), 147 (40), 218 (43), 233 (50); HRMS calcd for $\mathrm{C}_{13} \mathrm{H}_{20} \mathrm{BNO}_{2} ; 233.1587$ found; 233.1576.

3.2.12. 2-(1-Butyl)-4,4,5,5-tetramethyl-[1,3,2]-dioxaborolane (2l): ${ }^{1} \mathrm{H}$ NMR (400 $\left.\mathrm{MHz}, \mathrm{CDCl}_{3}\right) \delta 0.78(\mathrm{t}, J=7.7 \mathrm{~Hz}, 2 \mathrm{H}), 0.88(\mathrm{t}, J=7.2 \mathrm{~Hz}, 3 \mathrm{H}), 1.25(\mathrm{~s}, 12 \mathrm{H})$, 1.27-1.43 (m, 4H); ${ }^{13} \mathrm{C}$ NMR (100 MHz, $\left.\mathrm{CDCl}_{3}\right) \oint 13.8,24.7,25.3,26.1,82.7$; MS (EI) m/z 43 (16), 59 (24), 85 (50), 129 (62), 169 (100), 184 (3); HRMS calcd for $\mathrm{C}_{10} \mathrm{H}_{21} \mathrm{BO}_{2}$; 184.1635 found; 184.1638 .

3.2.13. 4,4,5,5-Tetramethyl-2-(3-phenyl-propyl)-[1,3,2]-dioxaborolane $\quad(2 \mathrm{~m}):{ }^{1} \mathrm{H}$ NMR (400 MHz, $\left.\mathrm{CDCl}_{3}\right) \delta .0 .83(\mathrm{t}, J=7.8 \mathrm{~Hz}, 2 \mathrm{H}), 1.24(\mathrm{~s}, 12 \mathrm{H}), 1.73(\mathrm{tt}, J=7.8,7.9$ $\mathrm{Hz}, 2 \mathrm{H}), 2.60(\mathrm{t}, J=7.9 \mathrm{~Hz}, 2 \mathrm{H}), 7.14-7.21(\mathrm{~m}, 3 \mathrm{H}), 7.24-7.26(\mathrm{~m}, 2 \mathrm{H}) ;{ }^{13} \mathrm{C} \mathrm{NMR}(100$ $\left.\mathrm{MHz}, \mathrm{CDCl}_{3}\right) \delta 24.8,26.1,38.6,82.9,125.5,128.1,128.5,142.7$; MS (EI) $\mathrm{m} / \mathrm{z} 41$ (22), 59 (10), 85 (100), 91 (62), 118 (93), 127 (24), 146 (13), 173 (12), 231 (11), 246 (32); HRMS calcd for $\mathrm{C}_{15} \mathrm{H}_{23} \mathrm{BO}_{2} ; 246.1791$ found; 246.1796.

3.2.14. 2-(Cyclohexyl)-4,4,5,5-tetramethyl-[1,3,2]-dioxaborolane (2n): ${ }^{1} \mathrm{H}$ NMR (400 $\left.\mathrm{MHz}, \mathrm{CDCl}_{3}\right) \delta$ 0.93-1.04 (m, 1H), $1.23(\mathrm{~s}, 12 \mathrm{H}), 1.26-1.36(\mathrm{~m}, 4 \mathrm{H}), 1.54-1.70(\mathrm{~m}, 6 \mathrm{H})$ ${ }^{13} \mathrm{C}$ NMR (100 MHz, $\left.\mathrm{CDCl}_{3}\right) \delta 24.7,26.7,27.1,28.0,82.7$; MS (EI) m/z 43 (23), 69 (44), 82 (30), 85 (26), 110 (30), 124 (100), 129 (23), 195 (38); HRMS calcd for $\mathrm{C}_{12} \mathrm{H}_{23} \mathrm{BO}_{2} ; 210.1791$ found; 210,1773. 
3.2.15. (Exo)-2-(Bicyclo-[2,2,1]-hept-2-yl)-4,4,5,5-tetrametyl-[1,3,2]-dioxaborolane (2o): ${ }^{1} \mathrm{H}$ NMR (400 MHz, $\left.\mathrm{CDCl}_{3}\right) \delta$ 0.87-0.94 (m, 1H), 1.23 (s, 12H), 1.14-1.40 (m, 4H), 1.42-1.68 (m, 4H), 2.22-2.29 (m, 2H); ${ }^{13} \mathrm{C}$ NMR (100 MHz, $\left.\mathrm{CDCl}_{3}\right) \delta 24.7,29.3,32.2$, 32.2, 36.6, 38.1, 38.7, 82.8; MS (EI) m/z 41 (100), 55 (42), 67 (34), 84 (33), 108 (12), 136 (14), 207 (15), 222 (0.6); HRMS calcd for $\mathrm{C}_{13} \mathrm{H}_{23} \mathrm{BO}_{2} ; 222.1791$ found; 222.1813.

3.2.16. 2-(4-tert-Butyl-cyclohexylmethyl)-4,4,5,5-tetramethyl-[1,3,2]-dioxaborolane (2p): ${ }^{1} \mathrm{H}$ NMR (400 MHz, $\left.\mathrm{CDCl}_{3}\right) ; \delta 0.69-1.02$ (m, 4H), 0.82 (s, 9H), 1.25 (s, 12H), $1.39-1.57(\mathrm{~m}, 5 \mathrm{H}), 1.69-1.78(\mathrm{~m}, 2 \mathrm{H}), 2.05(\mathrm{bs}, 1 \mathrm{H}) ;{ }^{13} \mathrm{C} \mathrm{NMR}\left(100 \mathrm{MHz}, \mathrm{CDCl}_{3}\right) \delta$ 21.2, 24.8, 27.5, 28.6, 32.8, 36.3, 48.6, 82.7; MS (EI) m/z 41 (27), 57 (61), 85 (100), 87 (24), 95 (24), 101 (55), 129 (39), 167 (24), 223 (20), 265 (13), 280 (11); HRMS calcd for $\mathrm{C}_{17} \mathrm{H}_{33} \mathrm{BO}_{2} ; 280.2574$ found; 280.2584 .

3.2.17. 2-[3-(tert-Butyldimethylsilyloxy)-2-methylpropyl]-4,4,5,5-tetramethyl[1,3,2]-dioxaborolane (2q): ${ }^{1} \mathrm{H}$ NMR $\left(400 \mathrm{MHz}, \mathrm{CDCl}_{3}\right) \delta 0.002(\mathrm{~s}, 6 \mathrm{H}), 0.55(\mathrm{dd}, J=$ 8.8, 15.6 Hz, 1H), 0.82-0.85 (m, 1H), $0.86(\mathrm{~s}, 9 \mathrm{H}), 0.87(\mathrm{~d}, J=6.5 \mathrm{~Hz}, 2 \mathrm{H}), 1.22(\mathrm{~s}$, $12 \mathrm{H}), 1.76-1.89(\mathrm{~m}, 1 \mathrm{H}), 3.28(\mathrm{dd}, J=7.2,9.6 \mathrm{~Hz}, 1 \mathrm{H}), 3.40(\mathrm{dd}, J=5.7,9.6 \mathrm{~Hz}, 1 \mathrm{H})$; ${ }^{13} \mathrm{C}$ NMR $\left(100 \mathrm{MHz}, \mathrm{CDCl}_{3}\right) \delta-5.35,18.3,19.0,24.8,26.0,32.2,70.0,82.8 ; \mathrm{MS}(\mathrm{EI})$ m/z 75 (24), 115 (23), 101 (11), 115 (23), 157 (100), 257 (23), 299 (2), 313 (0.2); HRMS calcd for $\mathrm{C}_{12} \mathrm{H}_{26} \mathrm{BO}_{3} \mathrm{Si}$ (- tert-butyl); 274.1744 found; 274.1753.

3.2.18. 2-(1,2-Dimethyl-propyl)-4,4,5,5-tetramethyl-[1,3,2]-dioxaborolane $(2 \mathrm{r}):{ }^{1} \mathrm{H}$ NMR (400 MHz, $\left.\mathrm{CDCl}_{3}\right) ; \delta 0.86(\mathrm{~d}, J=6.8 \mathrm{~Hz}, 6 \mathrm{H}), 0.90(\mathrm{~d}, J=6.6 \mathrm{~Hz}, 3 \mathrm{H}), 1.23-1.28$ $(\mathrm{m}, 1 \mathrm{H}), 1.25(\mathrm{~S}, 12 \mathrm{H}), 1.42-4.52(\mathrm{~m}, 1 \mathrm{H}) ;{ }^{13} \mathrm{C} \mathrm{NMR}\left(100 \mathrm{MHz}, \mathrm{CDCl}_{3}\right) \delta 11.7,22.2$, 24.8, 32.9, 82.8; MS (EI) m/z 41 (16), 57 (38), 69 (16), 83 (35), 87 (37), 99 (34), 129 (100), 183 (52), 198 (17); HRMS calcd for $\mathrm{C}_{11} \mathrm{H}_{23} \mathrm{BO}_{2}$; 198.1791 found; 198.1791. 
Keywords: Hydroboration, Pinacolborane, Iridium, Rhodium

*Corresponding author. Tel \& FAX: +81 11706 6561; e-mail address: miyaura@org-mc.eng.hokudai.ac.jp

\section{References and notes}

[1] (a) Onak, T. Organoborane Chemistry, Academic Press, New York, 1975; (b) Mikhailov, B. M.; Bubnov, Y. N. Organoboron Compounds in Organic Synthesis, Harwood Academic Pub., Amsterdam, 1983; (c) Pelter, A.; Smith, K.; Brown, H.

C. Borane Reagents, Academic Press, London, 1988; (d) Smith, K.; Pelter, A. Comprehensive Organic Synthesis, Trost, B. M.; Fleming, I. Eds., Pergamon Press, Oxford, 1991; Vol. 8, pp.703; (e) Matteson, D. Stereodirected Synthesis with Organoboranes, Springer, Berlin, 1995; (f) Vaultier, M.; Carboni, B. Comprehensive Organometallic Chemistry II, Abel, E. W.; Stone, F. G. A.; Wilkinson, G. Eds., Pergamon Press, Oxford, 1995; Vol. 11, pp.191.

[2] Männing, D.; Nöth, H. Angew. Chem. Int. Ed. Engl. 1985, 24, 878.

[3] (a) Brown, H. C.; Gupta, S. K. J. Am. Chem. Soc. 1971, 93, 1816. (b) Lane, C. F.; Kabalka, G. W. Tetrahedron 1976, 32, 981.

[4] Tucker, C. E.; Davidson, J.; Knochel, P. J. Org. Chem. 1992, 57, 3482.

[5] For reviews, see; (a) Burgess, K.; Ohlmeyer, M. J. Chem. Rev. 1991, 91, 1179. (b) Beletskaya, I.; Pelter, A. Tetrahedron 1997, 53, 4957. (c) Miyaura, N. Catalytic Hetero-Functionalization - From Hydroboration to Hydrozirconation, Togni, A.; Grützmacher, H. Eds., Wiley-VHC, Verlag Gmbh, Weinheim, 2001, p 1. 
[6] (a) Hayashi, T.; Matsumoto, Y.; Ito, Y. J. Am, Chem. Soc. 1989, 111, 3426. (b) Hayashi, T.; Matsumoto, Y. Ito, Y. Tetrahedron: Asymm. 1991, 2, 601.

[7] Westcott, S. A.; Blom, H. P.; Marder, T. B.; Baker, R. T. J. Am. Chem. Soc. 1992, $114,8863$.

[8] Burgess, K.; van der Donk, W. A.; Westcott, S. A.; Marder, T. B.; Baker, R. T.; Calabress, J. C. J. Am. Chem. Soc. 1992, 114, 9350.

[9] Westcott, S. A.; Marder, T. B.; Baker, R. T.; Calabrese, J. C. Can. J. Chem. 1993, $71,930$.

[10] Burgess, K.; Jaspers, M. Organometallics 1993, 12, 4197.

[11] (a) He, X.; Hartwig, J. F. J. Am. Chem. Soc. 1996, 118, 1696. (b) Hartwig, J. F.; Muhoro, C. N. Organometallics 2000, 19, 30.

[12] (a) Harrison, K. N.; Marks, T. J. J. Am. Chem. Soc. 1992, 114, 9220. (b) Bijpost, E. A.; Duchateau, R.; Teuben, J. H. J. Mol. Catal. A 1995, 95, 121.

[13] Pereira, S.; Srebnik, M. Tetrahedron Lett. 1996, 37, 3283. The authors have reported high yields and high level of terminal selectivities in hydroboration of both terminal and internal alkenes with pinacolborane in the presence of $\mathrm{Rh}\left(\mathrm{PPh}_{3}\right)_{3} \mathrm{Cl}$ or $\mathrm{Rh}(\mathrm{CO})\left(\mathrm{PPh}_{3}\right)_{2} \mathrm{Cl} .{ }^{13,16}$ However, we failed to reproduce the method when $\mathrm{Rh}\left(\mathrm{PPh}_{3}\right)_{3} \mathrm{Cl}$ and $\mathrm{Rh}(\mathrm{CO})\left(\mathrm{PPh}_{3}\right)_{2} \mathrm{Cl}$ were used.

[14] (a) Evans, D. A.; Fu, G. C.; Hoveyda, A. H. J. Am. Chem. Soc. 1988, 110, 6917. (b) Evans, D. A.; Fu, G. C.; Hoveyda, A. H. J. Am. Chem. Soc. 1992, 114, 6671.

[15] Evans, D. A.; Muci, A. R.; Stürmer, R. J. Org. Chem. 1993, 58, 5307.

[16] Pereira, S.; Srebnik, M. J. Am. Chem. Soc. 1996, 118, 909.

[17] Evans, D. A.; Fu, G. C.; Anderson, B. A. J. Am. Chem. Soc. 1992, 114, 6679. 
[18] (a) Brown, J. M.; Lloyd-Jones, G. C. J. Chem. Soc. Chem. Commun. 1992, 710. (b) Brown, J. M.; Lloyd-Jones, G. C. J. Am. Chem. Soc. 1994 116, 866.

[19] (a) Murata, M.; Watanabe, S.; Masuda, Y. Tetrahedron Lett. 1999, 40, 2585. (b) Murata, M.; Kawakita, K.; Asana, T.; Watanabe, S.; Masuda, Y. Bull. Chem. Soc. Jpn. 2002, 75, 825.

[20] Ramachandran, P. V.; Jennings, M. P.; Brown, H. C. Org. Lett. 1999, 1, 1399.

[21] (a) Osborn, J. A.; Jardine, F. H.; Young, J. F.; Wilkinson, G. J. Chem. Soc. A 1966, 1711. (b) Osborn, J. A.; Wilkinson, G. Inorg. Synth. 1967, 10, 67.

[22] (a) McCleverty, J. A.; Wilkinson, G. Inorg. Synth. 1968, 8, 214. (b) Evans, D.; Osborn, J. A.; Wilkinson, G. Inorg. Synth. 1968, 11, 99. (c) Ohgomori, Y.; Watanabe, Y. J. Chem. Soc. Dalton Trans. 1987, 2969.

[23] (a) Chat, J.; Venanzi, L. M. J. Chem. Soc. 1957, 4735. (b) Giordano, G.; Crabtree, H. Inorg. Synth. 1979, 19, 218.

[24] Green, M.; Kuc, T. A.; Taylor, S. H. J. Chem. Soc. A 1971, 2334.

[25] (a) Herde, J. L.; Lambert, J. C.; Senoff, C. V. Inorg. Synth. 1974, 15, 18. (b) Shenck, T. G.; Downs, J. M.; Milne, C. R. C.; Mackenzie, P. B.; Boucher, H.; Whealan, J.; Bosnich, B. Inorg. Chem. 1985, 24, 2334.

[26] (a) Haines, L. M.; Singleton, E. J. Chem. Soc. Dalton Trans. 1972, 1891. (b) Fordyce, W. A.; Crosby, G. A. Inorg. Chem. 1982, 21, 1455. 


$$
\begin{aligned}
& \mathrm{RCH}=\mathrm{CH}_{2}+\mathrm{H}^{-} \mathrm{B}_{\mathrm{O}}^{\mathrm{O}} \underset{\mathrm{O}}{\longrightarrow} \longrightarrow \text { Ir catalyst } \\
& 1 \text { HBpin }
\end{aligned}
$$

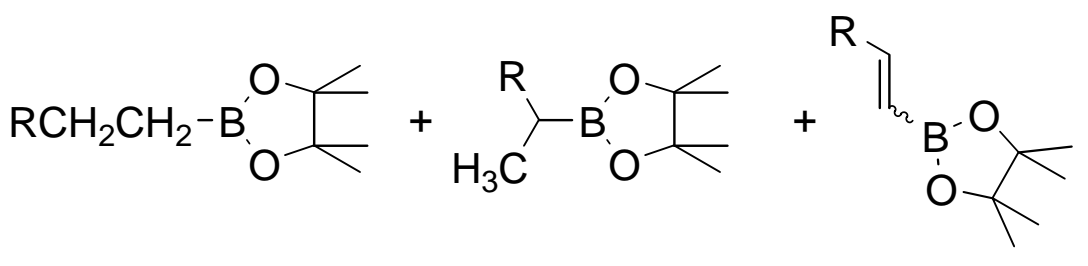


Table 1. Hydroboration of styrene with pinacolborane ${ }^{\mathrm{a}}$

\begin{tabular}{|c|c|c|c|c|c|}
\hline entry & catalyst & yield/ $/{ }^{b}$ & 2 & 3 & 4 \\
\hline 1 & $\mathrm{RhCl}\left(\mathrm{PPh}_{3}\right)_{3}$ & 26 & 66 & 34 & 4 \\
\hline 2 & $\mathrm{Rh}(\mathrm{CO})\left(\mathrm{PPh}_{3}\right)_{2} \mathrm{Cl}$ & 76 & 94 & 0 & 6 \\
\hline 3 & {$[\mathrm{Rh}(\mathrm{cod}) \mathrm{Cl}]_{2}$} & 48 & 75 & 2 & 23 \\
\hline 4 & $1 / 2[\mathrm{Rh}(\mathrm{cod}) \mathrm{Cl}]_{2} / \mathrm{dppm}$ & 74 & 29 & 47 & 24 \\
\hline 5 & 1/2[Rh(cod)Cl $]_{2} /$ dppe & 67 & 50 & 37 & 13 \\
\hline 6 & $1 / 2[\mathrm{Rh}(\mathrm{cod}) \mathrm{Cl}]_{2} / \mathrm{dppp}$ & 68 & 44 & 56 & 0 \\
\hline 7 & {$\left[\mathrm{Rh}(\mathrm{cod})_{2}\right] \mathrm{BF}_{4}$} & 28 & 42 & 32 & 26 \\
\hline 8 & {$\left[\mathrm{Rh}(\mathrm{cod})_{2}\right] \mathrm{BF}_{4} / \mathrm{dppm}$} & 73 & 38 & 42 & 20 \\
\hline 9 & {$\left[\mathrm{Rh}(\mathrm{cod})_{2}\right] \mathrm{BF}_{4} / \mathrm{dppe}$} & 79 & 37 & 56 & 7 \\
\hline 10 & {$\left[\mathrm{Rh}(\mathrm{cod})_{2}\right] \mathrm{BF}_{4} / \mathrm{dppb}$} & 51 & 39 & 31 & 30 \\
\hline 11 & {$[\mathrm{Ir}(\mathrm{cod}) \mathrm{Cl}]_{2}$} & 80 & 62 & 8 & 30 \\
\hline 12 & $1 / 2[\operatorname{Ir}(\operatorname{cod}) \mathrm{Cl}]_{2} / \mathrm{dppm}$ & 66 & 99 & 0 & 1 \\
\hline 13 & $1 / 2[\operatorname{Ir}(\mathrm{cod}) \mathrm{Cl}]_{2} / \mathrm{dppe}$ & 93 & 100 & 0 & 0 \\
\hline 14 & $1 / 2[\operatorname{Ir}(\operatorname{cod}) \mathrm{Cl}]_{2} / \mathrm{dppp}$ & 97 & 100 & 0 & 0 \\
\hline 15 & $1 / 2[\operatorname{Ir}(\operatorname{cod}) \mathrm{Cl}]_{2} / \mathrm{dppb}$ & 94 & 98 & 0 & 2 \\
\hline 16 & {$\left[\operatorname{Ir}(\operatorname{cod})_{2}\right] \mathrm{PF}_{6}$} & 19 & 67 & 11 & 22 \\
\hline 17 & {$\left[\operatorname{Ir}(\operatorname{cod})_{2}\left(\mathrm{PPh}_{3}\right)_{2}\right] \mathrm{PF}_{6}$} & 26 & 76 & 12 & 12 \\
\hline 18 & {$\left[\operatorname{Ir}(\mathrm{cod})_{2}\left(\mathrm{PMePh}_{2}\right)_{2}\right] \mathrm{PF}_{6}$} & $6 \quad 63$ & 100 & 0 & 0 \\
\hline 19 & {$\left[\operatorname{Ir}(\mathrm{cod})_{2}\right] \mathrm{PF}_{6} / 2 \mathrm{PCy}_{3}$} & 63 & 94 & 0 & 6 \\
\hline 20 & {$\left[\operatorname{Ir}(\operatorname{cod})_{2}\right] \mathrm{PF}_{6} / 2 \mathrm{P}^{t} \mathrm{Bu}_{3}$} & 46 & 80 & 7 & 13 \\
\hline 21 & {$[\operatorname{Ir}(\operatorname{cod})] \mathrm{PF}_{6} / \mathrm{dppm}$} & 63 & 96 & 0 & 4 \\
\hline 22 & {$\left[\operatorname{Ir}(\mathrm{cod})_{2}\right] \mathrm{PF}_{6} / \mathrm{dppe}$} & 12 & 24 & 41 & 35 \\
\hline 23 & {$\left[\operatorname{Ir}(\operatorname{cod})_{2}\right] \mathrm{PF}_{6} / \mathrm{dppp}$} & 26 & 60 & 13 & 27 \\
\hline 24 & {$\left[\operatorname{Ir}(\operatorname{cod})_{2}\right] \mathrm{PF}_{6} / \mathrm{dppb}$} & 25 & 67 & 12 & 21 \\
\hline
\end{tabular}

${ }^{a} \mathrm{~A}$ mixture of styrene $(1 \mathrm{mmol})$, pinacolborane $(1.2 \mathrm{mmol})$, catalyst (0.03 mmol based on the metals) in toluene was stirred for $24 \mathrm{~h}$ at room temperature.

${ }^{b}$ Isolated yields by chromatography. 
Table 2. Hydroboration of 1-octene with pinacolborane ${ }^{a}$

\begin{tabular}{clc}
\hline entry & catalyst & yield $/ \%^{b}$ \\
\hline 1 & $\mathrm{RhCl}\left(\mathrm{PPh}_{3}\right)_{3}$ & 18 \\
2 & $\mathrm{Rh}(\mathrm{CO})\left(\mathrm{PPh}_{3}\right)_{2} \mathrm{Cl}$ & 63 \\
3 & $1 / 2[\mathrm{Rh}(\mathrm{cod}) \mathrm{Cl}]_{2} / \mathrm{dppm}$ & 56 \\
4 & $1 / 2[\mathrm{Rh}(\mathrm{cod}) \mathrm{Cl}]_{2} / \mathrm{dppe}$ & 71 \\
5 & $1 / 2[\mathrm{Rh}(\mathrm{cod}) \mathrm{Cl}]_{2} / \mathrm{dppp}$ & 82 \\
6 & {$[\operatorname{Ir}(\mathrm{cod}) \mathrm{Cl}]_{2}$} & 50 \\
7 & $1 / 2[\operatorname{Ir}(\mathrm{cod}) \mathrm{Cl}]_{2} / 3 \mathrm{PCy}$ & 78 \\
8 & $1 / 2[\operatorname{Ir}(\mathrm{cod}) \mathrm{Cl}]_{2} / \mathrm{dppm}$ & 89 \\
9 & $1 / 2[\operatorname{Ir}(\mathrm{cod}) \mathrm{Cl}]_{2} / \mathrm{dppe}$ & 86 \\
10 & $1 / 2[\operatorname{Ir}(\mathrm{cod}) \mathrm{Cl}]_{2} / \mathrm{dppp}$ & 53 \\
11 & $1 / 2[\operatorname{Ir}(\mathrm{cod}) \mathrm{Cl}]_{2} / \mathrm{dppb}$ & 78 \\
\hline
\end{tabular}

${ }^{a} \mathrm{~A}$ mixture of 1 -octene $(1 \mathrm{mmol})$, pinacolborane $(1.2 \mathrm{mmol})$, and catalyst $\left(0.03 \mathrm{mmol}\right.$ based on the metals) in $\mathrm{CH}_{2} \mathrm{Cl}_{2}$ was stirred for $24 \mathrm{~h}$ at room temperature.

${ }^{b}$ Isolated yields of $\mathbf{2}$ by chromatography on silica gel. Formations of $\mathbf{3}$ and $\mathbf{4}$ were not observed. 
Table 3. Iridium-Catalyzed Hydroboration of Terminal Alkenes with Pinacolborane ${ }^{a}$

\begin{tabular}{|c|c|c|c|c|}
\hline entry & alkene & product & yield $/ \%^{b}$ & yield $/ \%^{b}$ \\
\hline 1 & $\mathrm{CH}_{3}\left(\mathrm{CH}_{2}\right)_{5} \mathrm{CH}=\mathrm{CH}_{2}$ & 2a & 89 & - \\
\hline 2 & $\mathrm{CH}_{3} \mathrm{C}(=\mathrm{O}) \mathrm{CH}_{2} \mathrm{CH}_{2} \mathrm{CH}=\mathrm{CH}_{2}$ & $I_{2} \quad \mathbf{2 b}$ & 68 & - \\
\hline 3 & $\mathrm{BrCH}_{2} \mathrm{CH}_{2} \mathrm{CH}=\mathrm{CH}_{2}$ & 2c & 77 & - \\
\hline 4 & $\mathrm{PhOCH}_{2} \mathrm{CH}=\mathrm{CH}_{2}$ & 2d & 89 & - \\
\hline 6 & $\mathrm{NCCH}_{2} \mathrm{CH}=\mathrm{CH}_{2}$ & $2 e$ & 15 & - \\
\hline 7 & $\mathrm{PhCH}=\mathrm{CH}_{2}$ & $2 f$ & 66 & 93 \\
\hline 9 & $4-\mathrm{CH}_{3} \mathrm{OC}_{6} \mathrm{H}_{4} \mathrm{CH}=\mathrm{CH}_{2}$ & $2 g$ & 76 & 80 \\
\hline 10 & 4- $\mathrm{CH}_{3} \mathrm{C}_{6} \mathrm{H}_{4} \mathrm{CH}=\mathrm{CH}_{2}$ & $2 h$ & 77 & 99 \\
\hline 11 & $\mathrm{C}_{6} \mathrm{~F}_{5} \mathrm{CH}=\mathrm{CH}_{2}$ & $2 \mathbf{i}$ & 60 & 82 \\
\hline 12 & 2-naphthylCH= $=\mathrm{CH}_{2}$ & $2 \mathbf{j}$ & 84 & 91 \\
\hline 13 & 4-pyridylCH= $\mathrm{CH}_{2}$ & $2 \mathbf{k}$ & 21 & - \\
\hline
\end{tabular}

${ }^{a}$ Alkene $(1 \mathrm{mmol})$ and pinacolborane $(1.2 \mathrm{mmol})$ were added to a solution of $[\mathrm{Ir}(\mathrm{cod}) \mathrm{Cl}]_{2}(0.015 \mathrm{mmol})$ and dppm or dppe $(0.03$ mmol) in $\mathrm{CH}_{2} \mathrm{Cl}_{2}$. The resulting mixture was stirred for $24 \mathrm{~h}$ at room temperature.

${ }^{b}$ Isolated yields of the terminal addition products (2) by Kugelrohr distillation or by chromatography over silica gel. The internal addition product (3) and the dehydrogenative coupling product (4) were less than $0.6 \%$ in each reactions. 


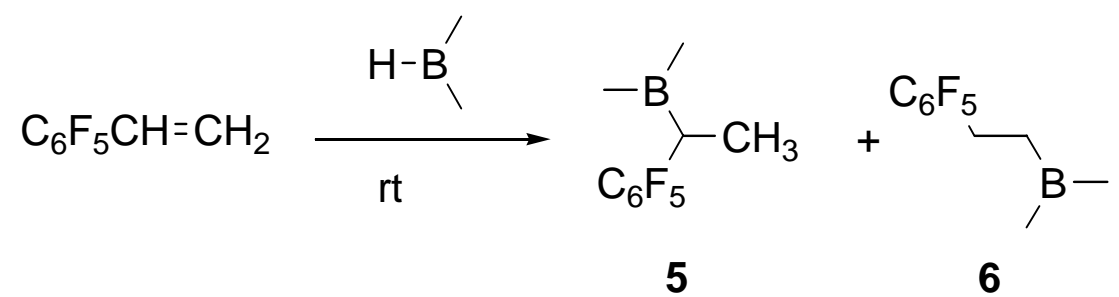

\begin{tabular}{llccc} 
borane & catalyst & $\mathbf{5 / \%}$ & $\mathbf{6 / \%}$ & ref \\
\hline HBcat & $\mathrm{RhCl}\left(\mathrm{PPh}_{3}\right)_{3}$ & 79 & 21 & {$[20]$} \\
HBpin & $\mathrm{RhCl}\left(\mathrm{PPh}_{3}\right)_{3}$ & 29 & 71 & {$[20]$} \\
HBpin & {$[\mathrm{IrCl}(\mathrm{cod})]_{2} / 2 \mathrm{dppe}$} & 0 & 100 & present
\end{tabular}


Table 4. Iridium-catalyzed hydroboration of internal alkenes with pinacolborane ${ }^{a}$

\begin{tabular}{clcc}
\hline entry & alkene & $\begin{array}{c}\text { product } \\
\text { No }\end{array}$ & \\
& & & \\
\hline & & $\mathbf{2 a}$ & $77^{c}$ \\
1 & $(E)$-4-octene & \\
2 & $(Z)-4-o c t e n e$ & $\mathbf{2 a}$ & $78^{c}$ \\
3 & $(Z)-\mathrm{CH}_{3} \mathrm{CH}=\mathrm{CHCH}_{3}$ & $\mathbf{2 l}$ & $65^{d}$ \\
4 & $(Z)-\mathrm{PhCH}^{b} \mathrm{CHCH}_{3}$ & $\mathbf{2 m}$ & $75^{e}$ \\
5 & cyclohexene & $\mathbf{2 n}$ & 74 \\
6 & norbornene & $\mathbf{2 o}$ & $66^{f}$ \\
7 & 1-t-butyl-4-methylenecyclohexane & $\mathbf{2 p}$ & 97 \\
8 & t-BuMe $2 \mathrm{SiOCH} \mathrm{C}_{2}\left(\mathrm{CH}_{3}\right)=\mathrm{CH}_{2}$ & $\mathbf{2 q}$ & 73 \\
9 & 2-methy-2-butene & $\mathbf{2 r}$ & $5(36)^{g}$ \\
\hline
\end{tabular}

${ }^{a} \mathrm{~A}$ mixture of alkene $(1 \mathrm{mmol})$, pinacolborane $(1.2 \mathrm{mmol})$, $[\operatorname{Ir}(\operatorname{cod}) \mathrm{Cl}]_{2}(0.015 \mathrm{mmol})$ and dppm $(0.03 \mathrm{mmol})$ in $\mathrm{CH}_{2} \mathrm{Cl}_{2}$ was stirred for $24 \mathrm{~h}$ at room temperature.

${ }^{b}$ Isolated yields.

${ }^{c}$ Pinacol 1-octylboronate.

${ }^{d}$ Pinacol 1-butylboronate. Dppb (0.03 mmol) was used in place of dppm.

${ }^{e}$ Pinacol 3-phenylpropylboronate.

${ }^{f}$ Exo isomer was selectively given.

${ }^{9}[\operatorname{Ir}(\operatorname{cod}) \mathrm{Cl}]_{2}(0.015 \mathrm{mmol})$ was used in the absence of phosphine ligand. 


$$
\begin{aligned}
(E)-\mathrm{C}_{3} \mathrm{H}_{7} \mathrm{CH}=\mathrm{CHC}_{3} \mathrm{H}_{7} & \stackrel{\text { 1. hydroboration }}{\text { 2. } \mathrm{H}_{2} \mathrm{O}_{2}, \mathrm{OH}^{-}} \\
& \text {1-octanol + 2-octanol + 3-octanol + 4-octanol }
\end{aligned}
$$

\begin{tabular}{llccccc} 
borane & catalyst & 1-ol & 2-ol & 3 -ol & 4 -ol & ref \\
\hline HBcat & $\mathrm{RhCl}_{\left(\mathrm{PPh}_{3}\right)_{3}}$ & 0 & 0 & 0 & 100 & {$[17]$} \\
$\mathrm{HBcat}$ & {$\left[\mathrm{Rh}(\mathrm{nbd})(\mathrm{dppb}) \mathrm{BF}_{4}\right.$} & 4 & 2 & 7 & 87 & {$[17]$} \\
$\left.\mathrm{HBpin}^{\mathrm{a}}\right)^{\mathrm{R}}$ & $\mathrm{RhCl}\left(\mathrm{PPh}_{3}\right)_{3}$ & 100 & 0 & 0 & 0 & {$[13,16]$} \\
HBpin $\left.^{\mathrm{a}}\right)$ & {$[\mathrm{IrCl}(\mathrm{cod})]_{2} / 2 \mathrm{dppp}$} & 100 & 0 & 0 & 0 & present
\end{tabular}

a) Isolated as the pinacol ester. 